\title{
Healthcare utilization and cost of cancer- related care prior to allogeneic hematopoietic cell transplantation for hematologic malignancies in the US: a retrospective real-world analysis
}

\author{
Machaon Bonafede ${ }^{1}$, Elias Anaissie ${ }^{2}$, Kristin Evans ${ }^{1}$ and Robbin Itzler ${ }^{3,4^{*}}$
}

\begin{abstract}
Background: Hematopoietic cell transplantation (HCT) is a potentially curative therapy as well as a costly procedure. Published studies have examined the cost of HCT in the US and the complications that follow but little is known about the cancer-related healthcare costs and resource utilization prior to the procedure and none of the studies have examined the variability in cost based on the type of hematologic malignancy involved. The aim of this study was to estimate mean cancer-related costs and resources incurred before the HCT is performed from the time the hematologic malignancy first develops.

Methods: The IBM ${ }^{\oplus}$ MarketScan ${ }^{\oplus}$ Research Databases were used to identify adult patients $\geq 18$ years of age with commercial or Medicare supplemental insurance who had undergone allogeneic HCT for hematologic malignancies from January 1, 2008 to December 31, 2017. Healthcare utilization and costs were assessed during the 6 months prior to diagnosis (pre-diagnostic period) and the follow-up period from diagnosis just prior to the HCT (pre-HCT period). Multivariable regression models were constructed to estimate total all-cause costs and cancer-related costs as well as healthcare utilization by type in each time period.

Results: A total of 2663 commercially insured patients and 266 with Medicare supplemental insurance were included in the study population. The mean-adjusted incremental cancer-related costs for commercially insured patients was $\$ 399,011$ in the overall observation period including the pre-diagnostic and pre-HCT periods combined, $9 \%$ of which was incurred in the pre-diagnostic period. The corresponding mean-adjusted incremental cancer-related costs for Medicare supplemental patients was $\$ 195,575$ for the same time period but the patterns of healthcare utilization were similar to the commercially insured population. Inpatient care accounted for approximately onehalf the cost in both patient populations. By type of hematologic malignancy, costs were lowest for myeloproliferative disorders $(\$ 211,561)$ and highest for acute lymphocytic leukemia $(\$ 462,072)$ in the commercially insured population.
\end{abstract}

\footnotetext{
* Correspondence: Robbin.Itzler@cslbehring.com

MB was employed at IBM Watson Health at the time the study was performed and is currently employed at Veradigm Life Sciences.

${ }^{3}$ CSL Behring, Marburg, Germany

${ }^{4}$ CSL Behring, King of Prussia, PA, USA

Full list of author information is available at the end of the article
}

C C The Author(s). 2021 Open Access This article is licensed under a Creative Commons Attribution 4.0 International License, which permits use, sharing, adaptation, distribution and reproduction in any medium or format, as long as you give appropriate credit to the original author(s) and the source, provide a link to the Creative Commons licence, and indicate if changes were made. The images or other third party material in this article are included in the article's Creative Commons licence, unless indicated otherwise in a credit line to the material. If material is not included in the article's Creative Commons licence and your intended use is not permitted by statutory regulation or exceeds the permitted use, you will need to obtain permission directly from the copyright holder. To view a copy of this licence, visit http://creativecommons.org/licenses/by/4.0/ The Creative Commons Public Domain Dedication waiver (http://creativecommons.org/publicdomain/zero/1.0/) applies to the data made available in this article, unless otherwise stated in a credit line to the data. 
Conclusion: This study demonstrates that overall patients with hematologic malignancies requiring HCT have considerable cancer-related healthcare resource utilization and costs leading up to HCT compared to the period of time prior to developing cancer.

Keywords: Hematologic malignancy, Hematopoietic cell transplantation, Claims-based study, Costs, Resource utilization

\section{Background}

The use of hematopoietic cell transplantation (HCT) for the treatment of hematologic malignancies is steadily rising; it is a potentially curative and vital therapy for many patients but is associated with a significant cost burden [1-4]. Studies conducted in the US to date have focused on the resource utilization and costs from the time of the HCT procedure and a time period following the procedure. Two studies limited the follow up period to the first 100 days after the procedure $[1,2]$. Two other studies followed patients for 1 year after the procedure [3, 4]. The high costs of HCT are partially related to post-HCT complications with previous studies showing higher healthcare costs in patients who developed complications compared with those who did not [4-6]. Only one study included cancer-related resource utilization and costs for a time period leading up to the HCT [3]. This study found that mean healthcare costs were $\$ 166,000$ higher in the 6 months leading up to the procedure compared to control patients with hematologic malignancies who did not receive an HCT. For patients who underwent allogeneic HCT, total costs in the 6 months before and 12 months after the procedure were $\$ 377,958$ higher than in control patients who did not undergo the procedure, with $82 \%$ of those costs being attributable to inpatient admissions and 15\% to outpatient services [5].

Although the aforementioned study provided valuable information about costs incurred around the time of the HCT, the study period did not fully capture all cancerrelated costs and healthcare utilization prior to the HCT. In addition, the patients with hematologic malignancies who undergo HCT differ from those patients who do not undergo HCT. For this reason, we chose to compare the costs of treatment before and after the cancer developed. To our knowledge, there is no existing literature describing cancer-related resource utilization for all time periods prior to the HCT.

The aim of this study was to estimate mean cancerrelated healthcare resource utilization and costs for all time periods prior to the HCT including the tests and procedures required to diagnose the hematologic malignancy. The cancer-attributable healthcare resource utilization and costs were determined by comparing the utilization and costs before and after the hematologic malignancy among patients who received an allogeneic HCT. This was to minimize the risk of bias that would occur by comparing those receiving an allogeneic HCT with those who do not. The study also aimed to identify factors that influence these cancer-related costs. The quantification of the costs attributable to cancer with the current standard of care is very important in economic assessments. It allows health economists to determine whether introducing a new therapy to treat a hematologic malignancy may improve health outcomes and reduce healthcare utilization that will offset the cost of introducing a new therapy.

\section{Methods}

\section{Data source}

Adults with commercial or Medicare supplemental insurance with a healthcare claim for an allogeneic HCT and a prior diagnosis of a hematologic malignancy were identified in the $\mathrm{IBM}^{\circ}$ MarketScan ${ }^{\circ}$ Research Databases.

\section{Study population}

Patients were included in the study if they had at least 18 months of continuous enrollment in MarketScan ${ }^{\circ}$ prior to their hematologic malignancy diagnosis, with no evidence of a prior HCT. Patients who met the following inclusion criteria were selected for the study: 1 ) a healthcare claim with a procedure code for an allogeneic HCT from January 1, 2008 to December 31, 2017; 2) at least one non-diagnostic medical claim with a diagnosis of at least one of the hematologic malignancies listed below prior to the date of the claim for HCT, as early as July 1 , $2005 ; 3$ ) at least 18 years old on the index date (date of the first hematologic malignancy diagnosis) and; 4) at least 18 months of continuous medical and prescription coverage prior to the index date.

Diagnostic claims for the following hematologic malignancy diagnoses were included in the analysis: acute lymphocytic leukemia (ALL), acute myelogenous leukemia (AML), chronic lymphocytic leukemia (CLL), chronic myelogenous leukemia (CML), Hodgkin's lymphoma, Non-Hodgkin's lymphoma (NHL), multiple myeloma (MM), myelodysplastic syndromes (MDS), myeloproliferative disorders (MPD; including myelofibrosis, polycythemia vera and essential thrombocythemia), and aplastic anemia (AA).

Patients with the following criteria were excluded from the study sample: 1 ) any evidence of an allogeneic HCT prior to the index date; 2) $\geq 3$ different hematologic 
malignancy diagnoses just prior to their HCT; 3) any of the following combinations of two different hematologic malignancy diagnoses just prior to their HCT: ALL + CLL, ALL + MM, AML + CLL, AML + Hodgkin's lymphoma, AML + MM, CLL + CML, CLL + MM and CML + $\mathrm{MM}$; 4) patients with paroxysmal nocturnal hemoglobinuria were excluded due to the small sample size. We assumed those patients with three or more different hematologic malignancy diagnoses just prior to their HCT or those with combinations of two different hematologic malignancies are therapy-related diseases, where they are diagnosed with one hematologic malignancy and then develop another therapy-related hematologic malignancy as a result of treatment for the first hematologic malignancy diagnosed. These patients were excluded because we thought the costs of these patients would differ from most patients who were diagnosed with one type of hematologic malignancy. Forty patients were excluded for having three or more different diagnoses and an additional 45 were excluded for having one of the following combinations of two diagnoses: ALL + CLL; ALL + MM; AML + CLL; AML + Hodgkin's; AML + MM; CLL + CML; CML + MM; CLL + MM.

\section{Study design}

The objective of the study was to compare the cancerrelated healthcare resource utilization and costs once the individuals develop cancer with the time period before they develop their cancer. The 6 months prior to the first cancer diagnosis was included in the observation period in order to capture the costs of tests and procedures required to diagnose the cancer. Therefore, the time period from 18 months to 6 months before the hematologic malignancy diagnosis was considered the baseline/control period, the 6-month period prior to the hematologic malignancy diagnosis was considered the pre-diagnostic period, and the time from the hematologic malignancy diagnosis to the HCT was the pre-HCT follow-up period (Fig. 1).
Healthcare resource utilization (inpatient [IP] admissions, emergency room [ER] visits, outpatient [OP] services, OP prescriptions) and costs were quantified during the baseline, pre-diagnostic, and pre-HCT followup periods, and the overall pre-HCT period which was defined as the pre-diagnostic period (6 months before the first hematologic malignancy diagnosis) and the preHCT follow-up (period of time from first hematologic malignancy diagnosis up until the HCT is performed). Cancer patients undergoing HCT were not compared with matched controls without cancer as it would be difficult to control for all the potential differences between these two groups of patients through propensity score matching. To ensure that all cancer-related costs were included, these were defined as the difference between baseline costs and pre-diagnostic as well as pre-HCT follow-up costs rather than specific diagnosis codes on the claims. This approach is more comprehensive than relying on specific cancer diagnosis codes and cancerrelated procedure codes because the immunosuppressive treatments that cancer patients receive often lead to infections and/or may interact with and exacerbate other comorbid conditions indirectly related to the cancer diagnoses. For the commercially insured population, separate healthcare resource utilization and cost analyses were conducted. In addition to analyzing the costs for all patients undergoing allogeneic HCTs regardless of type of hematologic malignancy, the healthcare resource utilization and cost for the five most common types of hematologic malignancies were analyzed separately. These analyses could not be performed in the Medicare supplemental population due to the small sample size.

Patients were classified into a malignancy cohort based on their hematologic malignancy diagnosis on the date of the HCT, or the closest date to the HCT where a hematologic malignancy diagnosis was observed on a claim.

\section{Statistical analysis}

Descriptive statistics were generated for categorical variables as the count and percentage of patients in each

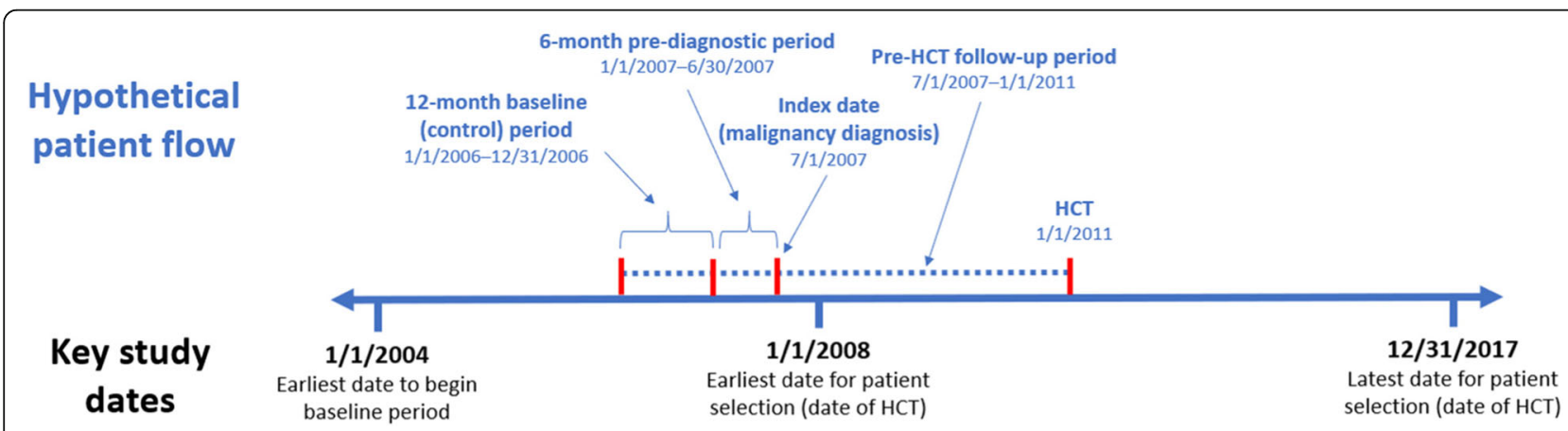

Fig. 1 Hypothetical patient flow. Full study period (1/1/2004-12/31/2017) and analysis dates and time periods for a hypothetical patient who had HCT on January 1, 2011 
category. Continuous variables were summarized by means, standard deviations and 95\% confidence intervals of the means and medians.

Multivariable regression models were constructed to estimate total all-cause costs in each time period to derive the cancer-related costs as well as different types of healthcare utilization. All-cause costs in the baseline period were subtracted from all-cause costs in the prediagnostic, pre-HCT, and the overall observation periods to determine patients' cancer-related costs in each of the time periods. Separate models were developed for the commercially insured and Medicare supplemental populations. Models for commercially insured patients controlled for type of hematologic malignancy, payer type, demographic characteristics (age, sex, geographic region, insurance plan coverage, year of diagnosis), clinical characteristics (comorbid disease and medication use throughout the study) and time to HCT. For the Medicare models, only patient demographics and time to HCT were included due to the small sample size. Adjusted cancer-related healthcare utilization by type of service was modelled only for the commercially insured population because the sample size for the Medicare supplemental population was deemed too small.

Distribution of the outcome determined the statistical approach. Separate piecewise gamma family generalized linear models (GLM) with a log link were used to estimate the cancer-related costs for the overall pre-HCT period. These models employed smoothed penalized splines by diagnosis group to address heterogeneity in costs by hematologic malignancy type. Subset analysis was conducted to evaluate costs by hematologic malignancy. Hurdle models were used to estimate hospitalization and ER visit costs. Covariate-adjusted counts of IP hospitalizations and ER visits were estimated using negative binomial GLM models, while hurdle models were used to estimate the count of OP visits for the commercially insured population. When models did not converge, a hurdle model with a Poisson distribution was used.

All costs are expressed in 2018 constant US dollars, adjusted using the Medical Care component of the Consumer Price Index (CPI).

\section{Results}

In total there were 9468 patients in the MarketScan ${ }^{\circ}$ commercial and Medicare Supplemental Databases with a code for an allogeneic HCT within the study period; 2929 met all inclusion/exclusion criteria and were included in the study (commercially insured: $N=2663$; Medicare supplemental: $N=266$ ). Patients' baseline characteristics are shown in Supplementary Table 1 (commercial) and 2 (Medicare supplemental). Sixty-four percent $(n=4185)$ of those excluded did not have $\geq 18$ months of observation time prior to their hematologic malignancy diagnosis. Another 23.2\% $(n=1518)$ did not have $\geq 1$ diagnostic medical claim with a diagnosis for a hematologic malignancy. The remaining $12.8 \%(n=836)$ were either not $\geq 18$ years of age, had $\geq 2$ different hematologic malignancy diagnoses just prior to their $\mathrm{HCT}$, or had $\mathrm{PNH}$, which was excluded from further analysis due to the small sample size.

Eighty-five percent of patients included in the study had at least one of the five main malignancy types: AML, NHL, MDS, ALL and MPD. The remaining 15\% of the population had CLL, HL, MM, or AA.

Overall, results for the Medicare supplemental population were comparable to those for the commercially insured population. As most patients included in the analysis were commercially insured, results are presented for the commercially insured population only unless otherwise stated.

\section{Time from hematologic malignancy diagnosis to HCT}

The mean (standard deviation [SD]) time from hematologic malignancy diagnosis to HCT was 12.3 (15.4) months for all patients, 12.5 (15.7) months for commercially insured patients and 10.5 (12.0) months for Medicare patients. The median time (range) from diagnosis to HCT was $6.3(0.0-131.1), 6.3$ (0.0-131.1) and $6.2(0.1-85.3)$ months for all patients, commercially insured and Medicare patients, respectively.

Among commercially insured patients, $48.3 \%$ had their HCT within 6 months of their initial hematologic malignancy diagnosis, $21.5 \%$ between 6 and 12 months after diagnosis and 30.2\% > 12 months after diagnosis; among Medicare supplemental patients, $48.8 \%$ had their HCT within 6 months after their initial hematologic malignancy diagnosis, $25.6 \%$ 6-12 months after diagnosis and $25.6 \%>12$ months after their initial diagnosis.

The mean time from hematologic malignancy diagnosis to HCT for commercially insured patients varied by the type of hematologic malignancy involved ranging from 8.1 months among ALL and AML patients, 12.4 and 14.5 months for MDS and MPD, respectively, and up to 21.0 months among NHL patients. Of ALL and AML patients, $\geq 60 \%$ received their $\mathrm{HCT}$ within 6 months of diagnosis while only $21.7 \%$ of NHL patients received their HCT within 6 months. (Table 1 ). The results were relatively similar for Medicare supplemental patients (data not shown).

\section{Total cancer-related healthcare costs}

The mean-adjusted incremental cancer-related cost for commercially insured patients was $\$ 399,011$ for the overall observation period including the pre-diagnostic and the pre-HCT periods combined. Approximately $9 \%$ 
Table 1 Time from initial hematologic malignancy diagnosis to HCT by type of hematologic malignancy. All commercially insured patients included. HCT, hematopoietic cell transplantation; SD, standard deviation

\begin{tabular}{|c|c|c|c|c|c|}
\hline \multirow[t]{2}{*}{ Diagnosis } & \multicolumn{2}{|c|}{ Months to HCT } & \multicolumn{3}{|c|}{$\begin{array}{l}\text { Proportion of patients with } \leq 6,>6 \text { and }<12 \text {, and } \geq 12 \text { months between their } \\
\text { initial diagnosis and HCT }\end{array}$} \\
\hline & Mean (SD) & Median & $\leq 6$ months & $>6$ and $<12$ months & $\geq 12$ months \\
\hline All patients $(N=2663)$ & $12.5(15.7)$ & 6.3 & $1287(48.3 \%)$ & $573(21.5 \%)$ & $807(30.2 \%)$ \\
\hline Acute lymphocytic leukemia $(N=346)$ & $8.1(10.0)$ & 4.9 & $210(60.7 \%)$ & $82(23.7 \%)$ & $54(15.6 \%)$ \\
\hline Acute myelogenous leukemia $(N=984)$ & $8.1(10.5)$ & 4.4 & $650(66.1 \%)$ & $156(15.9 \%)$ & $178(18.0 \%)$ \\
\hline Non-Hodgkin's lymphoma $(N=447)$ & $21.0(20.8)$ & 13.7 & $97(21.7 \%)$ & $105(23.5 \%)$ & $245(54.8 \%)$ \\
\hline Myelodysplastic syndrome $(N=330)$ & $12.4(17.4)$ & 6.4 & $154(46.7 \%)$ & $90(27.3 \%)$ & $86(26.0 \%)$ \\
\hline Myeloproliferative disorders $(N=148)$ & $14.5(18.6)$ & 7.8 & $61(41.2 \%)$ & $36(24.3 \%)$ & $51(34.5 \%)$ \\
\hline
\end{tabular}

of these incremental costs were incurred in the prediagnostic period, and the remainder were incurred in the subsequent pre-HCT follow-up period (i.e., the period from hematologic malignancy diagnosis up to HCT) (Fig. 2). The corresponding adjusted incremental cancer-related costs for Medicare supplemental patients was substantially lower at $\$ 195,575$ for the prediagnostic and pre-HCT periods combined.

The mean-adjusted cancer-related healthcare costs during the overall observation period among commercially insured patients by hematologic malignancy diagnosis are shown in Fig. 2. Mean cancer-related costs varied among the five main hematologic malignancies of interest, ranging from $\$ 211,561$ for MPD patients up to $\$ 462,074$ among patients with ALL for the overall observation period. As shown in Fig. 3, inpatient costs accounted for approximately half of the mean cancer-related costs in the overall observation period among the commercially insured population. OP interactions accounted for $28 \%$ of the cancerrelated costs and radiology services, lab services, and ER visits accounted for the remainder of the costs.

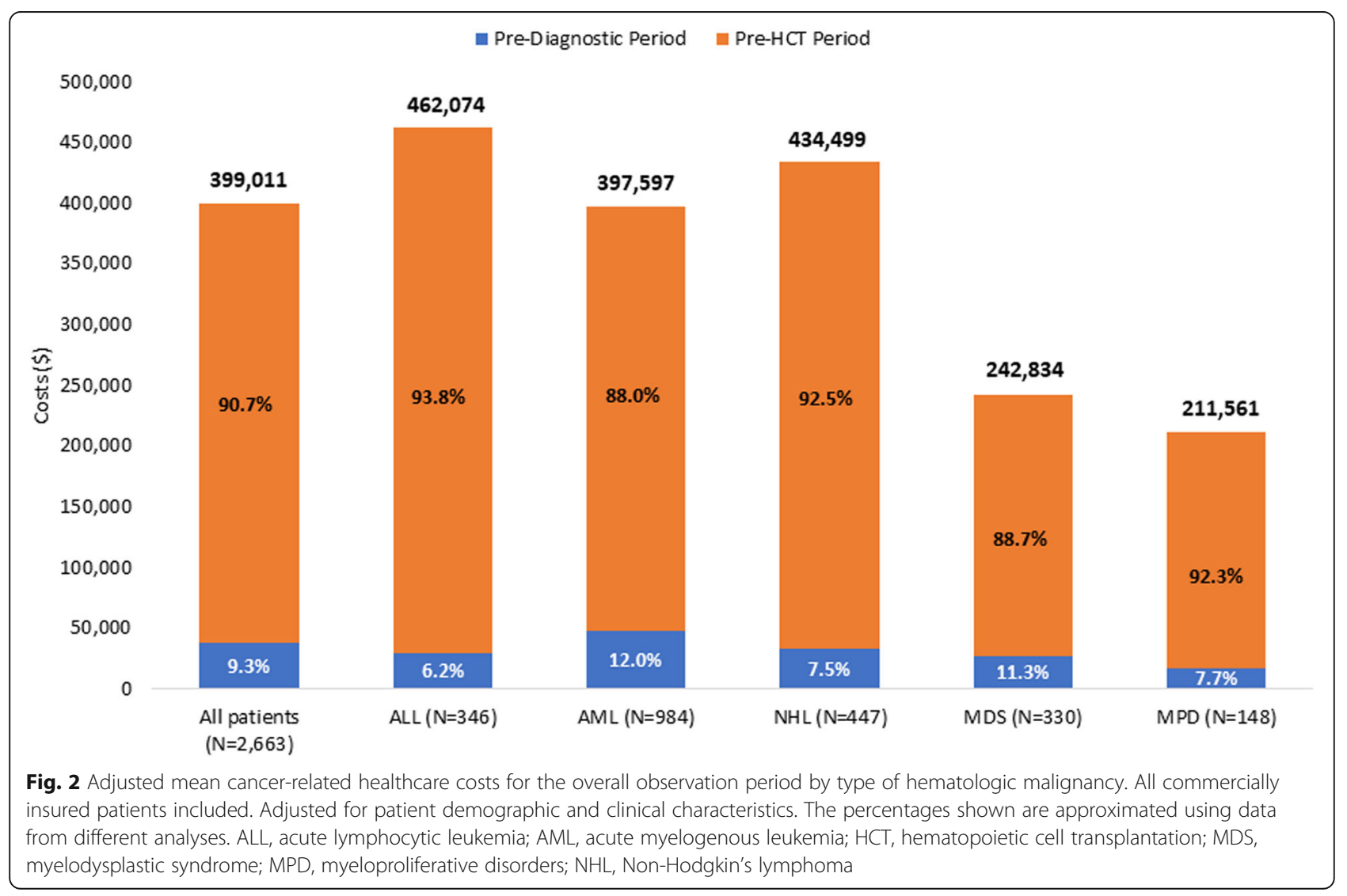




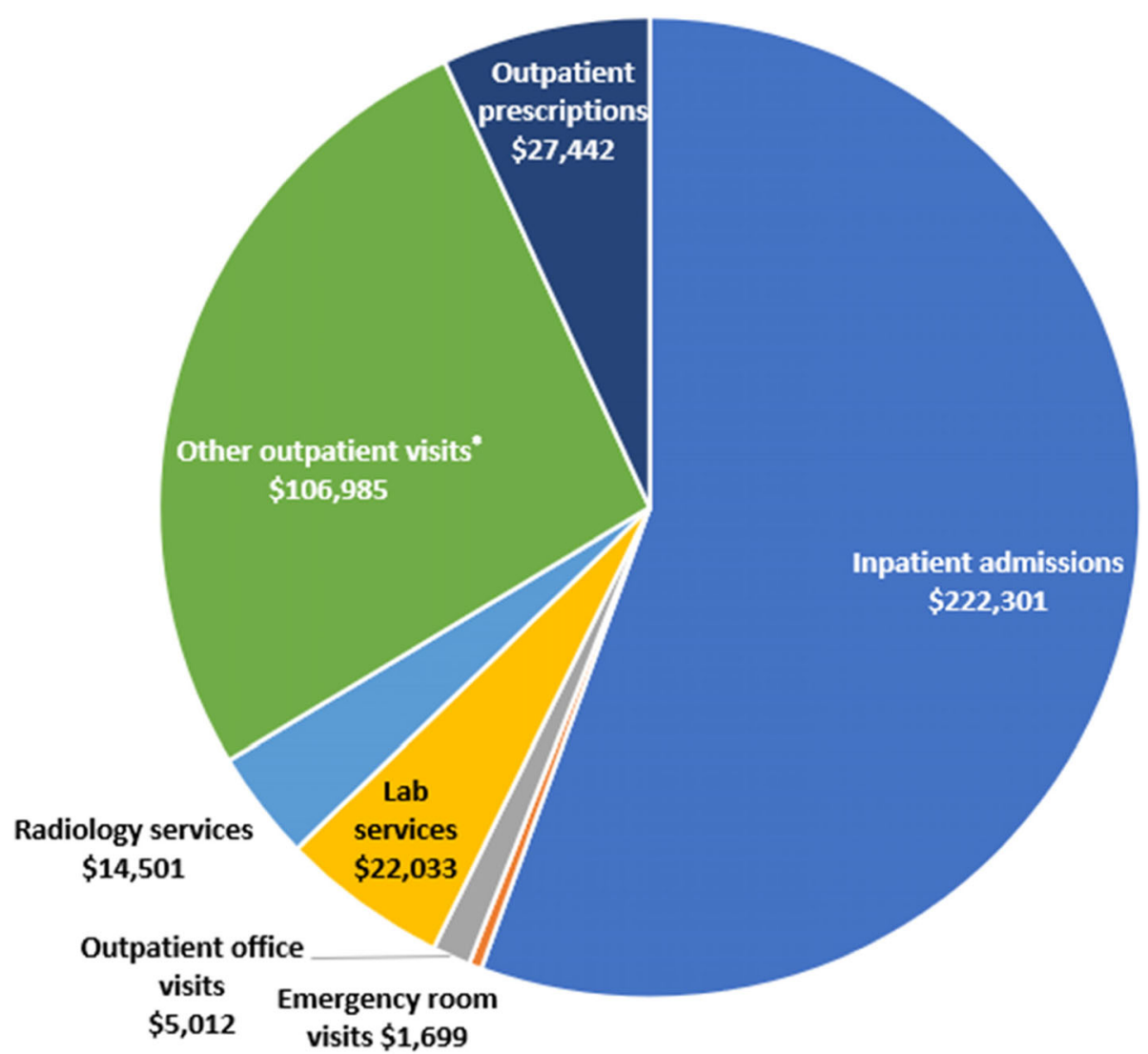

Fig. 3 Adjusted mean cancer-related healthcare costs, overall and by service type, during the overall observation period. All commercially insured patients included. Adjusted using the Medical Care component of the Consumer Price Index. Note that there are slight differences in the overall mean cost regardless of hematologic malignancy and the sum of the mean costs by service type due to the use of different modelling techniques to fit the data. ${ }^{*}$ Other outpatient visits include interactions not captured by office visits including home healthcare, walk-in health clinic services, and ambulatory surgical centers. HCT, hematopoietic cell transplantation

\section{Cancer-related healthcare utilization}

The adjusted number of cancer-related hospitalizations, ER visits, and OP visits by time period among all commercially insured patients are shown in Table 2. Eightyseven percent of commercially insured patients had at least one cancer-related hospitalization during the overall observation period, with an adjusted mean of 3.6 cancer-related hospitalizations. Fifty-six percent had at least one cancer-related ER visit (adjusted mean: 1.7 visits), and $96 \%$ had at least one cancer-related OP office visit (adjusted mean: 27.1 visits).

Separate healthcare utilization and cost multivariable models were developed for different hematologic malignancies among commercially insured patients so that

Table 2 Adjusted number of cancer-related hospitalizations, ER visits, and outpatient office visits by defined period. *OP office visits include only office visits and no other outpatient services. ER, emergency room; HCT, hematopoietic cell transplantation; OP, outpatient

\begin{tabular}{|c|c|c|c|}
\hline \multirow[b]{2}{*}{ Type of Cancer-Related Healthcare Interaction } & \multicolumn{3}{|c|}{$\begin{array}{l}\text { Commercially Insured Patients } \\
N=2663\end{array}$} \\
\hline & Pre-Diagnostic Period & Pre-HCT Follow-Up Period & Overall Observation Period \\
\hline$\geq 1$ cancer-related hospitalization $(\mathrm{N}, \%)$ & $690(25.9 \%)$ & $2170(81.5 \%)$ & $2311(86.8 \%)$ \\
\hline Number of hospitalizations & 0.43 & 3.21 & 3.61 \\
\hline$\geq 1$ cancer-related emergency room visit $(\mathrm{N}, \%)$ & $678(25.5 \%)$ & $1227(46.1 \%)$ & $1503(56.4 \%)$ \\
\hline Number of emergency room visits & 0.39 & 1.32 & 1.66 \\
\hline$\geq 1$ cancer-related OP office visit* $(\mathrm{N}, \%)$ & $2033(76.3 \%)$ & $2550(95.8 \%)$ & $2550(95.8 \%)$ \\
\hline Number of OP office visits & 6.69 & 30.53 & 27.10 \\
\hline
\end{tabular}


this information would be available to researchers developing economic evaluations for different purposes. Although the differences in cancer-related costs for different hematologic malignancies were not tested for statistical significance, we did observe that there were differences between the healthcare utilization, cancerrelated costs and timing of care across the five major hematologic malignancies. Among the five main diagnoses of interest, commercially insured ALL patients had the highest adjusted number of cancer-related hospitalizations (5.6) during the overall observation period, and MPD patients had the lowest (1.6); adjusted results were estimated using GLM including hematologic diagnoses, controlling for baseline hospitalizations (Table 3). The adjusted mean number of cancer-related ER visits was highest for commercially insured ALL patients (2.1) and lowest among AML patients (1.6). The adjusted mean number of cancer-related OP office visits ranged from 17.1 among AML patients, up to 23.3 among NHL patients.

Highest total cancer-related costs were observed for ALL and NHL (\$462,074 and $\$ 434,499$, respectively); costs for AML were somewhat lower $(\$ 397,597)$ while MDS and MPD were associated with relatively lower total cancer-related costs $(\$ 242,834$ and $\$ 211,561$, respectively) in the overall observation period. The higher costs among patients with NHL is best explained by the fact that NHL patients undergo several therapies before $\mathrm{HCT}$ including stem cell mobilization and autologous $\mathrm{HCT}$, in the event of relapse following several cycles of chemotherapy, and the course of the disease leading up to the need for an allogeneic HCT usually spans two to three years [7]. Indeed, NHL had the slowest progression to $\mathrm{HCT}$ with $21.7 \%$ progressing within 6 months while the other malignancies had $40-60 \%$ progressing to $\mathrm{HCT}$ within 6 months. The costs of NHL stand in sharp contrast to the costs for MDS and MPD, which are typically more indolent conditions, usually managed with relatively inexpensive oral or subcutaneous agents before progressing, in a relatively small proportion of patients, to require allogeneic HCT (Tables 1 and 3). This is also reflected by the lower likelihood of hospitalization among patients with MDS and MPD (Table 3). The higher intensity treatments typically given to treat ALL and AML also explain the higher costs incurred over a shorter period before HCT.

Notably, the cost of treatment per month until HCT was approximately $\$ 40,000$ for the entire study population and was highest for ALL and AML, approximately $\$ 57,000$ and $\$ 49,000$ per month respectively, and lowest for NHL, MDS and MPN (\$21,000, \$19,000, and \$15,000 per month, respectively).

The results of multivariable analyses for total cancerrelated costs during the diagnostic/pre-HCT period showed $13 \%$ greater adjusted costs for patients with their index diagnosis during 2010-2013 or during 2014-2017, compared to $2005-2009$ ( $p<0.05$ for both).

\section{Discussion}

In a retrospective claims-based analysis of 2663 patients with commercial insurance and 266 patients with Medicare supplemental insurance, mean cancer-related healthcare costs during the overall observation period were estimated at nearly $\$ 400,000$ for commercially insured patients, and nearly $\$ 200,000$ for Medicare supplemental patients, with nearly half of those costs incurred due to hospitalizations. Between 6 and 13\% (depending on type of hematologic malignancy) of the costs were in the pre-diagnostic period.

The patterns of resource utilization for the Medicare supplemental population were similar to the commercially insured population but the costs of care were lower due to lower rates of Medicare reimbursement. We also observed differences in healthcare utilization and cost between the different hematologic malignancies as well as the timing of care across the five major hematologic malignancies. Highest total cancer-related costs were observed for ALL and NHL (\$462,074 and $\$ 434,499$, respectively); cancer-related costs for AML were somewhat lower $(\$ 397,597)$ while MDS and MPD were associated with relatively lower total cancer-related costs ( $\$ 242,834$ and $\$ 211,561$, respectively) in the overall observation period. The differences in healthcare utilization and cost between the different hematologic malignancies are likely due to differences in the management of each malignancy and the position of allogeneic HCT in the therapeutic paradigm prior to HCT as well as the clinical course of each malignancy including

Table 3 Adjusted mean number of cancer-related hospitalizations, ER visits, and outpatient office visits by diagnosis. Overall observation period used stratified by the five main hematologic diagnoses of interest. ER, emergency room

\begin{tabular}{llll}
\hline & Hospitalizations & Emergency room visits & Outpatient office visits \\
\hline Acute lymphocytic leukemia $(N=346)$ & 5.62 & 2.14 & 18.30 \\
Acute myelogenous leukemia $(N=984)$ & 4.26 & 1.55 & 17.10 \\
Non-Hodgkin's lymphoma $(N=447)$ & 4.35 & 2.05 & 23.26 \\
Myelodysplastic syndrome $(N=330)$ & 2.00 & 1.74 & 20.18 \\
Myeloproliferative disorders $(N=148)$ & 1.62 & 1.75 & 19.67 \\
\hline
\end{tabular}


mortality rates, health outcomes, and speed of progression. The distribution of costs did not necessarily correlate with the length of time from diagnosis of the hematologic malignancy to the HCT procedure because of the factors mentioned above including the course (indolent versus rapid) and the type of therapies used for the various underlying diseases. The time from hematologic malignancy diagnosis to HCT varied from around 8 months for ALL and AML, around one year for MDS and MPD to almost two years for NHL.

A significant effect of more recent diagnosis (during 2010-2013 or 2014-2017 vs. 2005-2009) on cancerrelated costs is likely related to the novel therapies that became available, particularly for ALL during these separate study periods as well as the introduction of new diagnostics $[8,9]$. It is difficult to include all the advances in treatments that contributed to the changes in cost over time; however, we adjusted costs using the medical component of the CPI, which should help stabilize costs across eras.

Prior research focused on the high costs associated with HCT and its subsequent complications [4-6]. Only one study examined the cancer-related costs in the six months prior to the HCT [3]. The results of this study are consistent with the earlier research, but the cancerrelated costs reported prior to the HCT period are more comprehensive. Although the costs may differ depending on the type of underlying hematologic malignancy identified, the cancer-related costs are substantial for all types of hematologic malignancies. This earlier time period is vital to understanding the full breadth of HCTrelated healthcare resource utilization and costs.

The limitations of this study include those inherent to retrospective claims-based analyses. Detailed medical histories are not available so the variables that can be evaluated are limited. Also, the sample with Medicare supplemental insurance was small. As a result, many of the analyses are limited to only those individuals with commercial health coverage. Therefore, the results may not be generalizable to patients with other insurance or without health insurance. However, it is important to note that the patterns of healthcare utilization for the Medicare population were similar even though the rates of reimbursement were different. Moreover, there is a potential for misclassification of hematologic malignancies, allogeneic HCT or other variables and outcomes. The healthcare utilization and costs for new comorbid conditions after baseline were not identified separately but they were controlled for in the multivariable models. It is difficult to distinguish between conditions unrelated to the cancer diagnosis and those indirectly caused by the diagnosis of the hematologic malignancies. Lack of a diagnosis on a claim in the pre-index period does not necessarily mean the patient did not have the condition during baseline. Often after receiving a serious diagnosis such as cancer, a patient may have more frequent healthcare visits, and therefore pre-existing conditions may be identified as they are being diagnosed with a hematologic malignancy. Also, patients diagnosed with hematologic malignancies may require more medical resources for other comorbid conditions because of their cancer diagnosis and the fact that they are immunosuppressed. Despite these limitations, the majority of the patients undergoing $\mathrm{HCT}$ are commercially insured, the sample size is large and the time from onset of the hematologic malignancy to the HCT procedure is short so it is unlikely that the increase in comorbid conditions would have occurred in the absence of the cancer diagnosis. Finally, the costs for those who undergo HCT should not be compared to costs of those who do not undergo an HCT because these two groups of patients are not comparable with respect to their health status.

\section{Conclusions}

This analysis quantifies the cancer-related resources and costs incurred from the time patients develop their hematologic malignancy to the time they undergo an HCT. This analysis demonstrates that the cancer-related resources incurred from the time patients develop their hematologic malignancy to the time of the HCT are substantially and significantly higher than baseline costs for patients who progress to HCT. The cost estimates derived from these types of studies provide important information for decision making from an economic perspective.

\section{Abbreviations \\ AA: aplastic anemia; ALL: acute lymphocytic leukemia; AML: acute myelogenous leukemia; CLL: chronic lymphocytic leukemia; CML: chronic myelogenous leukemia; ER: emergency room; GLM: generalized linear models; HCT: hematopoietic cell transplantation; IN: inpatient; MDS: myelodysplastic syndromes; MM: multiple myeloma; MPD: myeloproliferative disorders; NHL: non-Hodgkin's lymphoma; OP: outpatient; PNH: paroxysmal nocturnal hemoglobinuria; SD: standard deviation; US: United States}

\section{Supplementary Information}

The online version contains supplementary material available at https://doi. org/10.1186/s12913-021-07150-4.

\section{Additional file 1.}

\section{Acknowledgements}

The authors wish to thank Stella Min for her contribution to this work. Editorial and medical writing support was provided by Meridian HealthComms Ltd., funded by CSL Behring.

Authors' contributions

$\mathrm{MB}, \mathrm{KE}, \mathrm{EA}$ and RI contributed equally to the study design, collection, analysis, interpretation of data, and the writing of this article. All authors reviewed the final manuscript and agreed on the decision to submit it for publication. 


\section{Funding}

This research was funded by CSL Behring. Editorial and medical writing support was provided by Meridian HealthComms Ltd., funded by CSL Behring.

\section{Availability of data and materials}

The datasets analyzed during the current study are not publicly available due to data use agreements with the underlying data contributors that restrict access to the data and prohibit the data from being publicly available. Data are available from the corresponding author on reasonable request.

\section{Declarations}

\section{Ethics approval and consent to participate}

Not applicable - our study used only de-identified data that was obtained from an existing database (IBM ${ }^{\circledR}$ MarketScan ${ }^{\oplus}$ Research) and did not involve the collection, use, or transmittal of individually identifiable data. All database records are statistically de-identified and certified to be fully compliant with US patient confidentiality requirements set forth in the Health Insurance Portability and Accountability Act of 1996 (HIPAA). As the database used in the study is fully de-identified and compliant with the HIPPA, this study was exempted from Institutional Review Board approval and informed consent was not needed. Permission to access the MarketScan ${ }^{\circledR}$ database was granted by Dan Barth-Jones (Columbia University).

\section{Consent for publication}

Not applicable - our study used only de-identified data obtained from an existing database and was compliant with the HIPPA, this study did not require patient consent for publication.

\section{Competing interests}

MB is an employee of Veradigm Life Sciences and was employed at IBM Watson Health at the time the study was performed. KE is an employee of IBM Watson Health. EA is an employee of CTI Clinical Trial and Consulting Services. $\mathrm{Rl}$ is an employee of CSL Behring.

\section{Author details}

${ }^{1}$ IBM Watson Health, Cambridge, MA, USA. ${ }^{2} \mathrm{CTI}$ Clinical Trial and Consulting Services, Covington, KY, USA. ${ }^{3}$ CSL Behring, Marburg, Germany. ${ }^{4}$ CSL Behring, King of Prussia, PA, USA.

Received: 11 March 2021 Accepted: 6 October 2021

Published online: 20 October 2021

\section{References}

1. Broder MS, Quock TP, Chang E, Reddy SR, Agarwal-Hashmi R, Arai S, et al. The cost of hematopoietic stem-cell transplantation in the United States. Am Health Drug Benefits. 2017;10(7):366-74.

2. Majhail NS, Mau LW, Denzen EM, Arneson TJ. Costs of autologous and allogeneic hematopoietic cell transplantation in the United States: a study using a large national private claims database. Bone Marrow Transplant. 2013;48(2):294-300. https://doi.org/10.1038/bmt.2012.133.

3. Bonafede M, Richhariya A, Cai Q, Josephson NC, McMorrow D, Garfin PM, et al. Real-world economic burden of hematopoietic cell transplantation among a large US commercially insured population with hematologic malignancies. J Med Econ. 2017;20(12):1244-51. https://doi.org/10.1080/13 696998.2017.1364648.

4. Svahn BM, Remberger M, Alvin O, Karlsson H, Ringden O. Increased costs after allogeneic haematopoietic SCT are associated with major complications and re-transplantation. Bone Marrow Transplant. 2012:47(5): 706-15. https://doi.org/10.1038/bmt.2011.162.

5. Yu J, Parasuraman S, Shah A, Weisdorf D. Mortality, length of stay and costs associated with acute graft-versus-host disease during hospitalization for allogeneic hematopoietic stem cell transplantation. Curr Med Res Opin. 2019:35(6):983-8. https://doi.org/10.1080/03007995.2018.1551193.

6. Grubb WW, Huse S, Alam N, Dychter S, Wingard JR, Majhail NS, et al. Economic Burden of Acute Graft-Versus-Host Disease (GvHD) Following Allogeneic Hematopoietic Cell Transplant (HCT) for Hematologic Malignancies. In: Economic burden of acute graft-versus-host disease (GvHD) following allogeneic hematopoietic cell transplant (HCT) for hematologic malignancies. DC: American Society of Hematology Washington; 2016.

7. Ansell SM. Symposium on neoplastic hematology and medical oncology non Hodgkin lymphoma: diagnosis and treatment. Mayo Clin Proc. 2015; 90(8):1152-63. https://doi.org/10.1016/j.mayocp.2015.04.025.

8. Rafei $\mathrm{H}$, Kantarjian HM, Jabbour EJ. Recent advances in the treatment of acute lymphoblastic leukemia. Leuk Lymphoma. 2019;60(11):2606-21. https://doi.org/10.1080/10428194.2019.1605071.

9. Zhao $Y$, Huang $H$, Wei GJ, et al. Novel Agents and Biomarkers for Acute Lymphoid Leukemia. Hematol Oncol. 2013;60(40).

\section{Publisher's Note}

Springer Nature remains neutral with regard to jurisdictional claims in published maps and institutional affiliations.
Ready to submit your research? Choose BMC and benefit from:

- fast, convenient online submission

- thorough peer review by experienced researchers in your field

- rapid publication on acceptance

- support for research data, including large and complex data types

- gold Open Access which fosters wider collaboration and increased citations

- maximum visibility for your research: over $100 \mathrm{M}$ website views per year

At $\mathrm{BMC}$, research is always in progress.

Learn more biomedcentral.com/submissions 